TRANSACTIONS OF THE

AMERICAN MATHEMATICAL SOCIETY

Volume 353, Number 3, Pages 877-891

S 0002-9947(00)02606-4

Article electronically published on November 14, 2000

\title{
CYCLIC COVERINGS AND HIGHER ORDER EMBEDDINGS OF ALGEBRAIC VARIETIES
}

\author{
THOMAS BAUER, SANDRA DI ROCCO, AND TOMASZ SZEMBERG
}

\begin{abstract}
In the present paper we study higher order embeddings in the context of cyclic coverings. Analyzing the positivity of the line bundle downstairs and its relationship with the branch divisor, we provide criteria for its pull-back to define an embedding of given order. We show that the obtained criteria are sharp. Finally, we apply them to various - sometimes seemingly unrelated-problems in algebraic geometry.
\end{abstract}

\section{INTRODUCTION}

In recent years there has been considerable interest in understanding under which circumstances linear series on algebraic varieties restrict surjectively to zerodimensional subschemes and to collections of fat points. Equivalently, one asks for the order of a given projective embedding of an algebraic variety. The concepts of higher order embeddings are captured by the notions of $k$-very ampleness, introduced in [6], and $k$-jet ampleness, studied in [9] and [15]. More geometrically, an algebraic variety embedded in $\mathbb{P}^{N}$ via a $k$-very ample line bundle has no $(k+1)$ secant $(k-1)$-plane $\mathbb{P}^{k-1} \subset \mathbb{P}^{N}$. The embedding given by a $k$-jet ample line bundle has stronger geometrical constraints related to the higher osculating planes.

By now, the situation on surfaces is quite well understood, mainly thanks to the availability of powerful methods such as a Reider type criterion for $k$-very ampleness 6] and the use of $\mathbb{Q}$-divisors in connection with the Kawamata-Viehweg vanishing theorem [20]. In higher dimensions, however, the problem seems to be much more difficult. While there are highly interesting general results on the separation of jets due to Demailly [15], there is still a lack of practical criteria that allows us to determine the order of a given embedding. The purpose of this paper is to study higher order embeddings of cyclic coverings $\pi: Y \longrightarrow X$, via line bundles given by pulling back "sufficiently positive" line bundles on $X$. Given a line bundle $L$ on $X$ we relate the order of the embedding defined by $\pi^{*} L$ to that of $L$ and certain rank one summands of the vector bundle $L \otimes \pi_{*} \mathcal{O}_{Y}$. The main results are expressed in the Theorems 2.1 and 3.1

In Section 1 we recall briefly the concepts of higher order embeddings. Section 2 is devoted to an exposition of the $k$-jet ample case. In Section 3 we prove our result concerning $k$-very ampleness. Although the proof is technically involved, we hope that the ideas behind are fairly transparent. Finally, the examples provided

Received by the editors February 28, 1999 and, in revised form, June 3, 1999.

2000 Mathematics Subject Classification. Primary 14C20.

The third author was supported by a research grant of Polish Academy of Sciences and KBN grant 2 P03A 00816. 
in the last section show that our results are sharp. In Proposition 4.6 we show how this type of result can be applied to the study of linear systems of plane curves.

We work throughout over the field $\mathbb{C}$ of complex numbers.

Acknowledgments. The first version of this paper was written during the authors' stay at the Max-Planck-Institut für Mathematik in Bonn. We would like to thank the Institute for financial support and for providing excellent working conditions. Our stay has been most fruitful and enjoyable.

\section{Preliminaries}

We start by recalling the notions of $k$-jet ampleness and $k$-very ampleness, which capture the concept of a higher order embedding in two different ways: the first notion requires the simultaneous separation of jets at finitely many points, whereas the second asks for the surjectivity of the restriction to 0-dimensional subschemes of certain length.

A line bundle $L$ on a smooth projective variety $X$ is called $k$-jet ample, if the evaluation map

$$
H^{0}(X, L) \longrightarrow H^{0}\left(X, L \otimes \mathcal{O}_{X} /\left(\mathfrak{m}_{y_{1}}^{k_{1}} \otimes \ldots \otimes \mathfrak{m}_{y_{r}}^{k_{r}}\right)\right)
$$

is surjective for any choice of distinct points $y_{1}, \ldots, y_{r}$ in $X$ and positive integers $k_{1}, \ldots, k_{r}$ with $\sum k_{i}=k+1$. Further, $L$ is called $k$-very ample, if for every zerodimensional subscheme $\left(\mathcal{Z}, \mathcal{O}_{Z}\right) \subset\left(X, \mathcal{O}_{X}\right)$ of length $\operatorname{dim}\left(H^{0}\left(Z, \mathcal{O}_{Z}\right)\right)=k+1$ the natural map

$$
H^{0}(X, L) \longrightarrow H^{0}\left(X, L \otimes \mathcal{O}_{Z}\right)
$$

is surjective.

As the definitions suggest, $k$-jet ampleness implies $k$-very ampleness [9, 2.2], and both notions are equivalent to global generation in the case $k=0$ and to very ampleness in the case $k=1$. In the case when $X=\mathbb{P}^{2}$, the notion of $k$-th order embedding is another way of dealing with "non-superabundant embeddings". We refer to [18] and [10] for more details.

For the purposes of the proof of Theorem 3.1 below, it will be useful to have a slightly more general definition of $k$-very ampleness: A subset $V \subset H^{0}(X, L)$ will be called $k$-very ample on an open subset $U \subset X$, if for every zero-dimensional subscheme $\left(\mathcal{Z}, \mathcal{O}_{\mathcal{Z}}\right) \subset\left(U, \mathcal{O}_{U}\right)$ of length $k+1$ the natural map

$$
\mathrm{ev}_{\mathcal{Z}}: V \longrightarrow H^{0}\left(X, L \otimes \mathcal{O}_{\mathcal{Z}}\right)
$$

is surjective. It is then obvious that if $V \subset H^{0}(X, L)$ is $k$-very ample on $U$, and if $\left(\mathcal{Z}, \mathcal{O}_{Z}\right) \subset\left(U, \mathcal{O}_{U}\right)$ is a 0 -dimensional subscheme of length $\ell$, then the affine subset

$$
\mathrm{ev}_{\mathcal{Z}}^{-1}(g) \subset V
$$

is $(k-\ell)$-very ample on $U \backslash \operatorname{supp}(\mathcal{Z})$ for every $g \in H^{0}\left(X, L \otimes \mathcal{O}_{\mathcal{Z}}\right)$.

\section{Separation of Simultaneous Jets}

In this section we prove:

Theorem 2.1. Let $X$ be a smooth projective variety and $B \subset X$ a smooth divisor. Let $M$ be a line bundle on $X$ such that $\mathcal{O}_{X}(d M) \cong \mathcal{O}_{X}(B)$ and let $\pi: Y \longrightarrow X$ be the cyclic covering of degree $d$ defined by $M$. Let $L$ be a line bundle on $X$ and $k$ a non-negative integer. If $L-q M$ is $(k-q)$-jet ample for $q=0, \ldots, \min (k, d-1)$, then $\pi^{*} L$ is $k$-jet ample. 
Proof. Let $\bar{M}$ be the total space of $M$ and $p: \bar{M} \longrightarrow X$ the bundle projection. Let $s_{B} \in H^{0}\left(X, \mathcal{O}_{X}(B)\right)$ be a section whose divisor of zeroes is $B$, and let $\tau \in$ $H^{0}\left(\bar{M}, p^{*} M\right)$ be the tautological section. As usual, $Y$ may be viewed as the divisor of zeroes of the section $p^{*} s_{B}-\tau^{d}$. Letting $t$ be the restriction of $\tau$ to $Y$ and $\pi: Y \longrightarrow X$ the restriction of $p$, the projection formula gives the decomposition

$$
H^{0}\left(Y, \pi^{*} L\right)=\bigoplus_{q=0}^{d-1} t^{q} \pi^{*} H^{0}(X, L-q M)
$$

corresponding to the eigenvalues of the action of the primitive covering automorphism $\varphi$ (see e.g. [1, Lemma I.17.2]).

Let $y_{1}, \ldots, y_{r} \in Y$ be points and $k_{1}, \ldots, k_{r}$ positive integers with $\sum k_{i}=k+1$. Given a simultaneous jet

$$
J \in \bigoplus_{i=1}^{r} H^{0}\left(Y, \pi^{*} L \otimes \mathcal{O}_{Y} / \mathfrak{m}_{y_{i}}^{k_{i}}\right)=H^{0}\left(Y, \pi^{*} L \otimes \mathcal{O}_{Y} / \mathfrak{m}_{y_{1}}^{k_{1}} \otimes \ldots \otimes \mathfrak{m}_{y_{r}}^{k_{r}}\right),
$$

we decompose $J$ into a sum of simultaneous jets $J=J_{1}+\ldots+J_{r}$, where $J_{i}=$ $\left(j_{1 i}, \ldots, j_{i i}, \ldots, j_{r i}\right)$, with $j_{l i}$ the zero jet of order $k_{l}$ at $y_{l}$ for $l \neq i$. It is enough to find sections $s_{i} \in H^{0}\left(Y, \pi^{*} L\right)$ such that

$$
s_{i} \bmod \mathfrak{m}_{y_{1}}^{k_{1}} \otimes \ldots \otimes \mathfrak{m}_{y_{r}}^{k_{r}}=J_{i} \quad \text { for } i=1, \ldots, r,
$$

since then the section $s=s_{1}+\ldots+s_{r}$ has the desired jet $J$. We may therefore assume $J=J_{1}$. The idea is now to construct such sections $s_{i}$ explicitly out of sections in the line bundles $L-q M, q \geq 0$. We distinguish three cases.

Case 1. Suppose first that $y_{1}$ is not contained in the ramification divisor $R$ of $\pi$, and that none of the points $y_{2}, \ldots, y_{r}$ lies in the orbit $\pi^{-1}\left(\pi\left(y_{1}\right)\right)$. This is the easiest case. The desired section is obtained as a pull-back of a section in $L$. More precisely we can view the jet $j_{11}$ as a jet at $\pi\left(y_{1}\right)$, via the isomorphism of local rings $\mathcal{O}_{Y, y_{1}} \cong \mathcal{O}_{X, \pi\left(y_{1}\right)}$ induced by $\pi$. (Here and in the sequel the term 'local ring' is meant with respect to the complex topology.) Since $L$ is $k$-jet ample, there is a section $s \in H^{0}(X, L)$ such that

$$
s \bmod \mathfrak{m}_{\pi\left(y_{1}\right)}^{k_{1}}=j_{11}
$$

and

$$
s \bmod \mathfrak{m}_{\pi\left(y_{i}\right)}^{k_{i}}=0 \quad \text { for } i=2, \ldots, r .
$$

Then of course $\pi^{*} s \bmod \mathfrak{m}_{y_{1}}^{k_{1}} \otimes \ldots \otimes \mathfrak{m}_{y_{r}}^{k_{r}}=J_{1}$.

Case 2. Suppose that $y_{1} \notin R, y_{1}, \ldots, y_{l}$ (with $l \geq 2$ ) lie in the orbit $\pi^{-1}\left(\pi\left(y_{1}\right)\right.$ ), and that the points $y_{l+1}, \ldots, y_{r}$ do not. This case is more difficult, since we have to separate points in a fiber of the covering. The construction of $s$ builds upon the cyclic group at hand. Let $k^{\prime}=\max \left(k_{1}, \ldots, k_{l}\right)$. Observe that

$$
k^{\prime} \leq k+1-(l-1)-\sum_{i=l+1}^{r} k_{i} .
$$

Let $\hat{j}_{11}$ be any preimage of the jet $j_{11}$ under the surjective map

$$
\mathcal{O} / \mathfrak{m}_{y_{1}}^{k^{\prime}} \longrightarrow \mathcal{O} / \mathfrak{m}_{y_{1}}^{k_{1}} \text {. }
$$


Again using the identification $\mathcal{O}_{Y, y_{1}} \cong \mathcal{O}_{X, \pi\left(y_{1}\right)}$, as well as the fact that $t\left(y_{1}\right) \neq 0$, for $0 \leq q \leq l-1$ we can find sections $s_{q} \in H^{0}(X, L-q M)$ such that

$$
\begin{aligned}
\pi^{*} s_{1} \bmod \mathfrak{m}_{y_{1}}^{k^{\prime}} & =\alpha_{1} \cdot \hat{j}_{11} \\
t \cdot \pi^{*} s_{2} \bmod \mathfrak{m}_{y_{1}}^{k^{\prime}} & =\alpha_{2} \cdot \hat{j}_{11} \\
\vdots & \\
t^{l-1} \cdot \pi^{*} s_{l} \bmod \mathfrak{m}_{y_{1}}^{k^{\prime}} & =\alpha_{l} \cdot \hat{j}_{11}
\end{aligned}
$$

and

$$
t^{q} \cdot \pi^{*} s_{q+1} \bmod \mathfrak{m}_{y_{i}}^{k_{i}}=0 \quad \text { for } 0 \leq q \leq l-1 \text { and } l+1 \leq i \leq r
$$

for any given complex numbers $\alpha_{1}, \ldots, \alpha_{l}$. The number $k^{\prime}+\sum_{i=l+1}^{r} k_{i}$ of conditions imposed on each $s_{q}$ does not exceed the positivity of $L-q M$ because of (2). Let $\varepsilon$ be a primitive $d$ th root of unity. Now, let $\left(\alpha_{1}, \ldots, \alpha_{l}\right)$ be a solution of the system of linear equations

$$
\begin{aligned}
\alpha_{1}+\ldots+\alpha_{l} & =1 \\
\alpha_{1}+\varepsilon^{\beta_{2}} \alpha_{2}+\ldots+\varepsilon^{(l-1) \beta_{2}} \alpha_{l} & =0 \\
& \vdots \\
\alpha_{1}+\varepsilon^{\beta_{l}} \alpha_{2}+\ldots+\varepsilon^{(l-1) \beta_{l}} \alpha_{l} & =0
\end{aligned}
$$

where $\beta_{2}, \ldots, \beta_{l}$ are integers such that $y_{i}=\varphi^{\beta_{i}}\left(y_{1}\right)$. (Note that the determinant of the system (3) is just the Vandermonde determinant of the numbers $1, \varepsilon^{\beta_{2}}, \ldots, \varepsilon^{\beta_{l}}$.) Consider the section $s=\pi^{*} s_{1}+\ldots+t^{l-1} \pi^{*} s_{l}$. We have $s \bmod \mathfrak{m}_{y_{1}}^{k^{\prime}}=\hat{j}_{11}$, which implies

$$
s \bmod \mathfrak{m}_{y_{1}}^{k_{1}}=j_{11},
$$

as required. Moreover, we have

$$
\begin{aligned}
s \bmod \mathfrak{m}_{y_{i}}^{k^{\prime}} & =\left(\varphi^{\beta_{i}}\right)^{*} s \bmod \mathfrak{m}_{y_{1}}^{k^{\prime}} \\
& =\pi^{*} s_{1}+\varepsilon^{\beta_{i}} t \pi^{*} s_{2}+\ldots+\varepsilon^{(l-1) \beta_{i}} t^{l-1} \pi^{*} s_{l} \bmod \mathfrak{m}_{y_{1}}^{k^{\prime}} \\
& =\left(\alpha_{1}+\varepsilon^{\beta_{i}} \alpha_{2}+\ldots+\left(\varepsilon^{\beta_{i}}\right)^{l-1} \alpha_{l}\right) \hat{j}_{11}=0,
\end{aligned}
$$

so that $s \bmod \mathfrak{m}_{y_{i}}^{k_{i}}=0$ for $i=2, \ldots, l$, and, of course,

$$
s \bmod \mathfrak{m}_{y_{i}}^{k_{i}}=0 \text { for } i=l+1, \ldots, r .
$$

Case 3. Finally, suppose that $y_{1} \in R$. Since $B$ (and hence $R$ ) is smooth, there are local coordinates $u_{1}, \ldots, u_{n}$ at $y_{1}$ and $v_{1}, \ldots, v_{n}$ at $\pi\left(y_{1}\right)$ such that $\pi$ is locally given as

$$
\pi\left(u_{1}, \ldots, u_{n}\right)=\left(u_{1}^{d}, u_{2}, \ldots, u_{n}\right)=\left(v_{1}, \ldots, v_{n}\right) .
$$

In these coordinates, the section $t$ is given by $u_{1}$ and $j_{11}$ may be written as

$$
\begin{aligned}
j_{11} & =\sum_{\substack{i_{1}+\ldots+i_{n} \leq k_{1}\\
}} a_{i_{1}, \ldots, i_{n}} u_{1}^{i_{1}} \cdot \ldots \cdot u_{n}^{i_{n}} \\
& =\sum_{l=0}^{d-1} u_{1}^{l} \sum_{\substack{i_{1} \equiv l \bmod d \\
i_{1}+\ldots+i_{n} \leq k_{1}}} a_{i_{1} \ldots i_{n}}\left(u_{1}^{d}\right)^{\frac{i_{1}-l}{d}} u_{2}^{i_{2}} \cdot \ldots \cdot u_{n}^{i_{n}} .
\end{aligned}
$$


This splitting reflects the fact that only jets containing powers of $u_{1}$ divisible by $d$ arise as pull-backs of jets on $X$. Since $L-q M$ is $(k-q)$-jet ample for $q=$ $0, \ldots, \min (d-1, k)$, there are sections $s_{q} \in H^{0}(X, L-q M)$ such that

$$
s_{q} \bmod \mathfrak{m}_{\pi\left(y_{1}\right)}^{k_{1}-q}=\sum_{\substack{i_{1} \equiv l \bmod d \\ \frac{i_{1}-q}{d}+i_{2}+\ldots+i_{n} \leq k_{1}-q}} a_{i_{1} \ldots i_{n}} v_{1}^{\frac{i_{1}-l}{d}} v_{2}^{i_{2}} \cdot \ldots \cdot v_{n}^{i_{n}},
$$

and

$$
s_{q} \bmod \mathfrak{m}_{\pi\left(y_{i}\right)}^{k_{i}}=0 \quad \text { for } i=2, \ldots, r .
$$

It is now easy to check that the section

$$
s=\sum_{q \geq 0} t^{q} \pi^{*} s_{q}
$$

has the prescribed jets.

Working as in Cases 1 and 2 of the above proof, one gets immediately the same result for unbranched coverings:

Theorem 2.2 (Unbranched case). Let $\pi: Y \longrightarrow X$ be an unbranched cyclic covering of degree $d$, defined by a line bundle $M$ with $\mathcal{O}_{X}(d M)=\mathcal{O}_{X}$. Let $L$ be a line bundle on $X$ and $k$ a non-negative integer. If $L-q M$ is $(k-q)$-jet ample for $q=0, \ldots, \min (k, d-1)$, then $\pi^{*} L$ is $k$-jet ample.

\section{Separation of zero-dimensional subschemes}

In Theorem 3.1 below, we will prove the $k$-very ampleness of the pull-back of an ample line bundle $L$ under suitable hypotheses on the line bundles $L-q M$, $q \geq 0$. In order to get a useful criterion, one needs to formulate a delicate numerical hypothesis. To this end, given positive integers $k$ and $\ell$, we introduce the abbreviations $\gamma(k, \ell)=1$ if $k / \ell$ is an integer, and $\gamma(k, \ell)=0$ otherwise, and we set

$$
\tau(k, \ell)=k-\left\lfloor\frac{k}{\ell}\right\rfloor-\ell+\gamma(k, \ell)+1 .
$$

Further, we use the notation

$$
\sigma(k, d, q)=\max \{\tau(k+1, \ell) \mid q+1 \leq \ell \leq \min (d, k+1)\}-1
$$

if $q>0$ and $\sigma(k, d, 0)=k$. An alternative definition of the function $\sigma$, which is useful for practical purposes, will be given in Remark 3.3

Our result can then be stated as follows.

Theorem 3.1. Let $X$ be a smooth projective variety and $B \subset X$ a smooth divisor. Assume that $M$ is a line bundle on $X$ such that $\mathcal{O}_{X}(d M) \cong \mathcal{O}_{X}(B)$, and let $\pi$ : $Y \longrightarrow X$ be the cyclic covering of degree d defined by $M$.

Let $L$ be a line bundle on $X$ and $k$ a non-negative integer. If $L-q M$ is $\sigma(k, d, q)$ very ample for $q=0, \ldots, \min (k, d-1)$, then $\pi^{*} L$ is $k$-very ample.

One can prove the $k$-very ampleness of $\pi^{*} L$ under the assumption that $L-q M$ is $(k-q)$-very ample for $0 \leq q \leq \min (k, d+1)$ by arguments very similar to the ones used in the proof of Theorem 2.1 The point, however, here is that $k$-very ampleness holds already under the much weaker (but also more intricate) hypotheses involving the numbers $\sigma(k, d, q)$. For instance, if $L$ is 2-very ample, then $\pi^{*} L$ is also 2 -very 
ample as soon as both $L-M$ and $L-2 M$ are globally generated. If $\pi$ is of degree 2 , it is enough to check that $L-M$ is globally generated. We provide a list of explicit values of $\sigma(k, d, q)$ in Remark [3.3. We have relegated the statement and proof of three technical lemmas needed in the proof of Theorem[3.1 to an appendix.

Proof of Theorem 3.1. Let $\left(\mathcal{Z}, \mathcal{O}_{\mathcal{Z}}\right) \subset Y$ be a zero-dimensional subscheme of length $k+1$, defined by the ideal $\mathcal{I}_{Z}$, and supported on the points $y_{1}, \ldots, y_{r}$. Let $k_{i}=$ length $\left(\mathcal{O}_{\mathcal{Z}, y_{i}}\right)$. Given an element

$$
g=\left(g_{1}, \ldots, g_{r}\right) \in \bigoplus_{i=1}^{r} H^{0}\left(\pi^{*} L \otimes \mathcal{O}_{\mathcal{Z}, y_{i}}\right)=H^{0}\left(\pi^{*} L \otimes \mathcal{O}_{\mathcal{Z}}\right)
$$

we will construct a section $s \in H^{0}\left(Y, \pi^{*} L\right)$ whose image in $H^{0}\left(\pi^{*} L \otimes \mathcal{O}_{\mathcal{Z}}\right)$ is $g$.

The strategy here is different from the proof of Theorem 2.1. Roughly speaking, for $q \geq 0$, we will approximate $s$ by building a sequence of affine subsets

$$
\cdots \subset V_{2}^{q} \subset V_{1}^{q} \subset V_{0}^{q} \subset H^{0}(X, L-q M)
$$

consisting of sections $s_{q, i} \in H^{0}(X, L-q M)$ such that the length of the subscheme of $\mathcal{Z}$ where $\sum t^{q} \pi^{*} s_{q, i} \bmod \mathcal{I}_{\mathcal{Z}}$ agrees with $g$ increases with $i$ reaching length $\mathcal{Z}$ in the last step. First, we take care of subschemes of $\mathcal{Z}$ supported in the ramification locus of $\pi$. Starting with a fiber containing most of the points $y_{i}$, we deal then with subschemes of $\mathcal{Z}$ supported in regular fibers of $\pi$. Each step consists now of fixing sections in $V_{i}^{q}$ for $q$ appropriately big and imposing new conditions on sections in $V_{i}^{q}$ for lower $q$ thus defining subsets $V_{i+1}^{q}$. The positivity assumptions of the theorem guarantee that we never run out of sections.

Let us now turn to the details. After reordering the points $y_{1}, \ldots, y_{r}$, we may assume that

$$
\begin{aligned}
y_{1}, \ldots, y_{n_{1}-1} & \in R \\
y_{n_{1}}, \ldots, y_{n_{2}-1} & \in \pi^{-1}\left(x_{1}\right) \\
& \vdots \\
y_{n_{m}}, \ldots, y_{n_{m+1}-1}=y_{r} & \in \pi^{-1}\left(x_{m}\right)
\end{aligned}
$$

for distinct points $x_{1}, \ldots, x_{n} \in X \backslash B$. Letting $l_{i}=n_{i+1}-n_{i}$, we may also assume that $l_{1} \leq \cdots \leq l_{m}$ and $k_{n_{i}}=\max \left(k_{n_{i}}, \ldots, k_{n_{i+1}-1}\right)$. Thus there are $l_{i}$ points in the fiber over $x_{i}$ and the point in the fiber with the lowest index is the "fattest" one. We will use the abbreviations $\mathcal{I}_{i}=\mathcal{I}_{\mathcal{Z}, y_{i}}$ for $i=1, \ldots, r$ and $K_{i}=k_{n_{i}}+\cdots+k_{n_{i+1}-1}$ for $i=1, \ldots, m$.

Identifying the local rings of the points in the same fiber by means of $\pi$, we consider the ideals

$$
\mathcal{J}_{i}=_{\text {def }} \mathcal{I}_{n_{i}+1} \cap \cdots \cap \mathcal{I}_{n_{i+1}-1} \subset \mathcal{O}_{Y, y_{n_{i}}}
$$

and for $i=1, \ldots, m$ we denote by

$$
\rho_{i}: \mathcal{O}_{Y, y_{n_{i}}} /\left(\mathcal{I}_{n_{i}} \cap \mathcal{J}_{i}\right) \longrightarrow \mathcal{O}_{Y, y_{n_{i}}} / \mathcal{J}_{i}
$$

and

$$
\eta_{i}: \mathcal{O}_{Y, y_{n_{i}}} /\left(\mathcal{I}_{n_{i}} \cap \mathcal{J}_{i}\right) \longrightarrow \mathcal{O}_{Y, y_{n_{i}}} / \mathcal{I}_{n_{i}}
$$

the quotient maps. Applying Lemma A.1, we get the inequalities 


$$
\operatorname{length}\left(\mathcal{O}_{X, x_{i}} / \mathcal{J}_{i}\right) \leq \tau\left(K_{i}, l_{i}\right)
$$

for all $i \in\{1, \ldots, m\}$ with $l_{i} \geq 2$.

First, we take care of points on the ramification divisor. Let $y_{i}$ be one of the points $y_{1}, \ldots, y_{n_{1}-1}$ on the ramification divisor $R$, and let $\mathcal{W}_{i}$ be the restriction of $\mathcal{Z}$ to $y_{i}$. Choosing coordinates $u_{1}, \ldots, u_{n}$ at the point $y_{i}$ as in Case 3 of the proof of Theorem 2.1, we have a decomposition

$$
\mathcal{I}_{\mathcal{W}_{i}}=\bigoplus_{q=0}^{d-1} u_{1}^{q} \pi^{-1} \mathfrak{a}_{i, q}
$$

where the $\mathfrak{a}_{i, q}$ are ideals in $\mathcal{O}_{X, \pi\left(y_{i}\right)}$. We observe that the non-trivial part of this decomposition sits in the range $0 \leq q \leq \min \left(d-1, k_{i}\right)$. So we get an isomorphism

$$
\mathcal{O}_{\mathcal{W}_{i}} \cong \bigoplus_{q=0}^{d-1} \mathcal{O}_{X} / \mathfrak{a}_{i, q} .
$$

Note that $\mathfrak{a}_{i, q} \subset \mathfrak{a}_{i, q+1}$ for $q=0, \ldots, d-2$, giving a cofiltration

$$
\mathcal{O}_{X} / \mathfrak{a}_{i, 0} \rightarrow \mathcal{O}_{X} / \mathfrak{a}_{i, 1} \rightarrow \cdots \rightarrow \mathcal{O}_{X} / \mathfrak{a}_{i, d-1} \rightarrow 0 .
$$

This implies length $\left(\mathcal{O}_{X} / \mathfrak{a}_{i, q}\right) \leq\left\lfloor\frac{k_{i}}{q+1}\right\rfloor$. Under the isomorphism (5)), the element $g_{i}$ corresponds to a $d$-tuple $\left(g_{i, 0}, \ldots, g_{i, d-1}\right)$ for $i=1, \ldots, n_{1}-1$. Since $L-q M$ is $\sigma(k, d, q)$-very ample, the subset

$$
V_{0}^{q}=\left\{s \in H^{0}(X, L-q M) \mid s \bmod \mathfrak{a}_{i, q}=g_{i, q} \text { for } i=1, \ldots, n_{1}-1\right\}
$$

is at least $\sigma_{0}(k, d, q)$-very ample on $X \backslash R$, where

$$
\sigma_{0}(k, d, q)=\sigma(k, d, q)-\sum_{i=1}^{n_{1}-1}\left\lfloor\frac{k_{i}}{q+1}\right\rfloor
$$

for $q=0, \ldots, \min (k, d-1)$. Lemma $\mathrm{A.3}$ assures that $\sigma_{0}(k, d, q)$ is non-negative and, in particular, implies that $V_{0}^{q}$ is non-empty. For any section $s$ of the form

$$
s=\pi^{*} s_{0}+t \pi^{*} s_{1}+\cdots+t^{\min (k, d-1)} \pi^{*} s_{\min (k, d-1)}
$$

with $s_{q} \in V_{0}^{q}$ we then have

$$
s \bmod \mathcal{I}_{i}=g_{i} \quad \text { in } \mathcal{O}_{Y, y_{i}}
$$

for $i=1, \ldots, n_{1}-1$. This completes the construction for the points on the ramification locus.

From now on we proceed in $m$ steps. The recursive procedure works so that the $i$-th step takes care of what we generate in the fiber over $x_{m-i+1}$.

Step 1 . For every $q \geq l_{m}$ such that $V_{0}^{q} \neq H^{0}(X, L-q M)$ we fix a section $s_{q} \in V_{0}^{q}$. For $i=1, \ldots, l_{m}$ we denote by $h_{i}$ the image

$$
\left(\varphi^{\beta\left(n_{m}, n_{m}+i-1\right)}\right)^{*}\left(\sum_{q \leq l_{m}} t^{q} \pi^{*}\left(s_{q}\right)\right) \bmod \mathcal{I}_{n_{m}} \cap \cdots \cap \mathcal{I}_{n_{m+1}-1}
$$

in the local ring $\mathcal{O}_{Y, y_{n_{m}}}$. We choose preimages $\hat{g}_{i}$ of $g_{n_{m}+i-1}$ in $\mathcal{O}_{Y, y_{n_{m}}} / \mathcal{I}_{n_{m}} \cap \cdots \cap$ $\mathcal{I}_{n_{m+1}-1}$. For $q \leq l_{m}-1$, we let $V_{1}^{q}$ be the subset of $V_{0}^{q}$ of sections $s_{q}$ satisfying 
the following conditions in $\mathcal{O}_{Y, y_{n_{m}}}$ :

(6)

$$
\begin{aligned}
t^{l_{m}-1} \pi^{*} s_{l_{m}-1} \bmod \mathcal{J}_{m} & =\alpha_{l_{m}-1,1} \rho_{m}\left(\hat{g}_{1}-h_{1}\right)+\cdots+\alpha_{l_{m}-1, l_{m}} \rho_{m}\left(\hat{g}_{l_{m}}-h_{l_{m}}\right) \\
& \vdots \\
t \pi^{*} s_{1} \bmod \mathcal{J}_{m} & =\alpha_{1,1} \rho_{m}\left(\hat{g}_{1}-h_{1}\right)+\cdots+\alpha_{1, l_{m}} \rho_{m}\left(\hat{g}_{l_{m}}-h_{l_{m}}\right) \\
\pi^{*} s_{0} \bmod \mathcal{I}_{n_{m}} \cap \mathcal{J}_{m} & =\alpha_{0,1}\left(\hat{g}_{1}-h_{1}\right)+\cdots+\alpha_{0, l_{m}}\left(\hat{g}_{l_{m}}-h_{l_{m}}\right),
\end{aligned}
$$

where $\alpha_{i, j}$ are solutions of the Vandermonde type systems of linear equations

$$
\begin{aligned}
\alpha_{0, i}+\cdots+\alpha_{l_{m}-1, i} & =\delta_{1 i} \\
\alpha_{0, i}+\varepsilon^{\beta\left(n_{m}, n_{m}+1\right)} \alpha_{1, i}+\cdots+\varepsilon^{\beta\left(n_{m}, n_{m}+1\right)} \alpha_{l_{m}-1, i} & =\delta_{2 i} \\
\alpha_{0, i}+\varepsilon^{\left(l_{m}-1\right) \beta\left(n_{m}, n_{m+1}-1\right)} \alpha_{1, i}+\cdots+\varepsilon^{\left(l_{m}-1\right) \beta\left(n_{m}, n_{m+1}-1\right)} \alpha_{l_{m}-1, i} & =\delta_{l_{m} i} .
\end{aligned}
$$

We observe that (4) implies that $V_{1}^{q}$ remains at least $\sigma_{1}(k, d, q)$-very ample, where for $q \geq 1$,

$$
\sigma_{1}(k, d, q)=\sigma_{0}(k, d, q)-\tau\left(K_{m}, l_{m}\right)
$$

and for $q=0$,

$$
\sigma_{1}(k, d, 0)=\sigma_{0}(k, d, 0)-\sum_{i=n_{m}}^{n_{m+1}-1} K_{i}=\sum_{i=1}^{n_{m}-1} k_{i} .
$$

Let $s$ be of the form $\pi^{*} s_{0}+\cdots+t^{l} \pi^{*} s_{l}$, where $s_{q} \in V_{1}^{q}$, for $q \leq l_{m}-1$. Then in the local ring $\mathcal{O}_{Y, y_{n}+i-1}$ we have

$$
\begin{aligned}
s \bmod \mathcal{I}_{n_{m}+i-1} & =\left(\varphi^{\beta\left(n_{m}, n_{m}+i-1\right)}\right)^{*} s \bmod \mathcal{I}_{n_{m}} \\
& =\left(\varphi^{\beta\left(n_{m}, n_{m}+i-1\right)}\right)^{*}\left(\pi^{*} s_{0}+\cdots+t^{l} \pi^{*} s_{l}\right) \bmod \mathcal{I}_{n_{m}} .
\end{aligned}
$$

Applying the definition of $\varphi$ we get the relation

$$
\begin{aligned}
s \bmod & \mathcal{I}_{n_{m}+i-1} \\
= & \pi^{*} s_{0}+\varepsilon^{\beta\left(n_{m}, n_{m}+i-1\right)} t \pi^{*} s_{1}+\cdots+\varepsilon^{\left(l_{m}-1\right) \beta\left(n_{m}, n_{m}+i-1\right)} t^{l_{m}-1} \pi^{*} s_{l_{m}-1} \\
& +\left(\varphi^{\beta\left(n_{m}, n_{m}+i-1\right)}\right)^{*}\left(\sum_{q \geq l_{m}} s_{q}\right) \bmod \mathcal{I}_{n_{m}} .
\end{aligned}
$$

Taking (6) and the definition of $\eta_{m}$ into account we obtain

$$
\begin{aligned}
s \bmod & \mathcal{I}_{n_{m}+i-1} \\
= & \alpha_{0,1} \eta_{m}\left(\hat{g}_{1}-h_{1}\right)+\cdots+\alpha_{0, l_{m}} \eta_{m}\left(\hat{g}_{l_{m}}-h_{l_{m}}\right)+\cdots+ \\
& +\varepsilon^{\beta\left(n_{m}, n_{m}+i-1\right)}\left(\alpha_{1,1} \eta_{m}\left(\hat{g}_{1}-h_{1}\right)+\cdots+\alpha_{1, l_{m}} \eta_{m}\left(\hat{g}_{l_{m}}-h_{l_{m}}\right)\right) \\
& \vdots \\
& \quad+\varepsilon^{\left(l_{m}-1\right) \beta\left(n_{m}, n_{m}+i-1\right)}\left(\alpha_{l_{m}-1,1} \eta_{m}\left(\hat{g}_{1}-h_{1}\right)+\cdots+\alpha_{l_{m}-1, l_{m}} \eta_{m}\left(\hat{g}_{l_{m}}-h_{l_{m}}\right)\right) \\
& +\eta_{m}\left(h_{i}\right) .
\end{aligned}
$$

Collecting the terms $\eta_{m}\left(\hat{g}_{i}-h_{i}\right)$ for $i=1, \ldots, l_{m}$, we obtain as coefficients terms satisfying (17) and finally arrive at

$$
s \bmod \mathcal{I}_{n_{m}+i-1}=g_{n_{m}+i-1}
$$

as desired. 
Step $i$. Proceeding as in Step 1 , we fix sections $s_{q} \in V_{i-1}^{q}$ for $l_{m-i+1} \leq q<l_{m-i+2}$ and we construct subspaces

$$
V_{i}^{q} \subset V_{i-1}^{q}
$$

for $q<l_{m-i+1}$, such that for all $s_{q} \in V_{i}^{q}$ one has

$$
\sum_{q=0}^{l} t^{q} \pi^{*} s_{q} \bmod \mathcal{I}_{j}=g_{j}
$$

for all $n_{m-i+1} \leq j<n_{m-i+2}$. Then $V_{i}^{q}$ is still $\sigma_{i}(k, d, q)$-very ample, where by (4)

$$
\sigma_{i}(k, d, q)=\sigma_{i-1}(k, d, q)-\tau\left(K_{i}, l_{i}\right)=\sigma_{0}(k, d, q)-\sum_{\nu=i+1}^{m} \tau\left(K_{\nu}, l_{\nu}\right)
$$

for $q \geq 1$ and

$$
\sigma_{i}(k, d, 0)=\sigma_{0}(k, d, 0)-K_{i}=\sum_{\nu=1}^{n_{m-i+1}-1} k_{\nu}
$$

After $m-1$ steps, by Lemma A.2, the space $V_{m-1}^{q}$ is still $\sigma_{m-1}(k, d, q)$-very ample with

$$
\sigma_{m-1}(k, d, q) \geq \tau\left(K_{1}, l_{1}\right)
$$

for $1 \leq q<l_{1}$ and

$$
\sigma_{m-1}(k, d, 0) \geq K_{1}
$$

This guarantees that we can find sections $s_{1}, \ldots, s_{l_{1}}$ such that

$$
s={ }_{\text {def }} \sum_{q=0}^{l} t^{q} \pi^{*} s_{q}
$$

satisfies $s \bmod \mathcal{I}_{\mathcal{Z}}=g$. This completes the proof of the theorem.

As in section 2 we get immediately the same result for unbranched coverings.

Theorem 3.2. Let $X$ be a smooth projective variety. Let $M$ be a line bundle on $X$ such that $\mathcal{O}_{X}(d M) \cong \mathcal{O}_{X}$ and let $\pi: Y \longrightarrow X$ be the cyclic covering of degree $d$ defined by $M$. Let $L$ be a line bundle on $X$ and $k$ a non-negative integer. If $L-q M$ is $\sigma(k, d, q)$-very ample for $q=0, \ldots, \min (k, d-1)$, then $\pi^{*} L$ is $k$-very ample.

Remark 3.3. For concrete applications of Theorem 3.1 one needs to compute the numbers $\sigma(k, d, q)$ appearing in the hypothesis there. We found the following alternative formula useful for practical purposes:

$$
\sigma(k, d, q)=\left\{\begin{aligned}
k \quad \text { if } q=0, \\
k+1-\left\lfloor\frac{k+1}{\lfloor\sqrt{k+1}\rfloor}\right\rfloor-\lfloor\sqrt{k+1}\rfloor+\gamma(k+1,\lfloor\sqrt{k+1}\rfloor) \\
\text { if } 1 \leq q<\lfloor\sqrt{k+1}\rfloor, \\
k-q-\left\lfloor\frac{k+1}{q+1}\right\rfloor+\gamma(k+1, q+1) \quad \text { if }\lfloor\sqrt{k+1}\rfloor \leq q .
\end{aligned}\right.
$$

We can omit the elementary proof.

Now, in order to convey some feeling for the numbers $\sigma(k, d, q)$, we include a table for $d=15$ and $0 \leq k \leq 15$. (The $k$-th column lists the values $\sigma(k, 15, q)$ for $0 \leq q \leq k$.) For instance, $\pi^{*} L$ is 2 -very ample if $L$ is so and both $L-M$ and 
TABLE 1. Values of the function $\sigma(k, d, q)$ for $d=15$ and $0 \leq k \leq 15$

\begin{tabular}{lrrrrrrrrrrrrrrrr}
\hline$k$ & 0 & 1 & 2 & 3 & 4 & 5 & 6 & 7 & 8 & 9 & 10 & 11 & 12 & 13 & 14 & 15 \\
\hline$L$ & 0 & 1 & 2 & 3 & 4 & 5 & 6 & 7 & 8 & 9 & 10 & 11 & 12 & 13 & 14 & 15 \\
$L-1 M$ & & 0 & 0 & 1 & 1 & 2 & 2 & 3 & 4 & 4 & 5 & 6 & 6 & 7 & 8 & 9 \\
$L-2 M$ & & & 0 & 0 & 1 & 2 & 2 & 3 & 4 & 4 & 5 & 6 & 6 & 7 & 8 & 9 \\
$L-3 M$ & & & & 0 & 0 & 1 & 2 & 3 & 3 & 4 & 5 & 6 & 6 & 7 & 8 & 9 \\
$L-4 M$ & & & & & 0 & 0 & 1 & 2 & 3 & 4 & 4 & 5 & 6 & 7 & 8 & 8 \\
$L-5 M$ & & & & & & 0 & 0 & 1 & 2 & 3 & 4 & 5 & 5 & 6 & 7 & 8 \\
$L-6 M$ & & & & & & & 0 & 0 & 1 & 2 & 3 & 4 & 5 & 6 & 6 & 7 \\
$L-7 M$ & & & & & & & 0 & 0 & 1 & 2 & 3 & 4 & 5 & 6 & 7 \\
$L-8 M$ & & & & & & & & 0 & 0 & 1 & 2 & 3 & 4 & 5 & 6 \\
$L-9 M$ & & & & & & & & & 0 & 0 & 1 & 2 & 3 & 4 & 5 \\
$L-10 M$ & & & & & & & & & & 0 & 0 & 1 & 2 & 3 & 4 \\
$L-11 M$ & & & & & & & & & & & 0 & 0 & 1 & 2 & 3 \\
$L-12 M$ & & & & & & & & & & & & 0 & 0 & 1 & 2 \\
$L-13 M$ & & & & & & & & & & & & & 0 & 0 & 1 \\
$L-14 M$ & & & & & & & & & & & & & & & & \\
\hline
\end{tabular}

$L-2 M$ are globally generated, and it is 4 -very ample if $L$ is so and $L-M$ and $L-2 M$ are very ample and $L-3 M$ and $L-4 M$ are globally generated.

\section{EXAMPLES AND APPLICATIONS}

This section serves two purposes. First, we give a number of examples showing that the assumptions in our results cannot be dropped. Secondly, we provide an application to the study of linear series of plane curves.

Theorems [2.1] and [3.1] state, roughly speaking, that under suitable assumptions on the bundles $L-q M$ the positivity of a pull-back $\pi^{*} L$ is at least as high as the positivity of $L$. It is then natural to ask whether the converse statement is also true. The following example shows that this is not the case:

Example 4.1. Let $(X, L)$ be a principally polarized abelian variety. A $d$-torsion point $M \in P i c^{0}(X)$ gives rise to a cyclic covering $\pi: Y \longrightarrow X$ such that $\left(Y, \pi^{*} L\right)$ is an abelian variety of type $(1, \ldots, 1, d)$. If $d$ is sufficiently large, then $\pi^{*} L$ will be very ample. (One knows by work of Debarre-Hulek-Spandaw 13 that it is enough to take $d>2^{g}$.) But $L$ is not even globally generated. In the surface case, where the generation of jets and the $k$-very ampleness is well understood (see [3]), one can even give explicit values $d_{k}$ (resp. $d_{k}^{\prime}$ ) for every $k>0$ such that $\pi^{*} L$ is $k$-jet ample (resp. $k$-very ample) whenever $d \geq d_{k}$ (resp. $d \geq d_{k}^{\prime}$ ).

The previous example shows that the positivity can very well increase after taking a pull-back. In general, however, it does not need to increase at all, as the following example shows:

Example 4.2. Consider a product of elliptic curves $X=E_{1} \times \cdots \times E_{n}, n \geq 2$, and the product polarization

$$
L=\mathcal{O}_{X}\left(\sum_{i=1}^{n} p r_{i}^{*}(0)\right),
$$

where $p r_{i}$ is the $i$-th projection. The choice of a $d$-torsion point on $E_{n}$ determines a covering $\tilde{E}_{n} \longrightarrow E_{n}$, which in turn induces a cyclic covering $Y=E_{1} \times \cdots \times E_{n-1} \times$ 
$\tilde{E}_{n} \longrightarrow X$ such that

$$
\pi^{*} L=\mathcal{O}_{Y}\left(\sum_{i=1}^{n-1} p r_{i}^{*}(0)+d \cdot p r_{n}^{*}(0)\right) .
$$

The line bundles $\mathcal{O}_{X}((k+2) L)$ and $\mathcal{O}_{Y}\left((k+2) \pi^{*} L\right)$ are then $k$-jet ample (by [2]), but neither of them is $\ell$-jet ample (or even $\ell$-very ample) for any $\ell>k$, since the restricted bundles $\mathcal{O}_{X}((k+2) L) \mid E_{1}$ and $\mathcal{O}_{Y}\left((k+2) \pi^{*} L\right) \mid E_{1}$ are only of degree $k+2$ (cf. [8, Proposition 1.4]).

Even if $L$ is positive, the positivity of $\pi^{*} L$ may drop if the assumptions on the line bundles $L-q M$ are not satisfied. The following example illustrates their importance.

Example 4.3. Let $X \cong \mathbb{P}^{n}$ and let $r$ and $d$ be integers greater than or equal to 2. Let $M=\mathcal{O}_{\mathbb{P}^{n}}(r)$ and let $B$ be a smooth member of $|d M|$. Let $\pi: Y \longrightarrow X$ be the cyclic covering defined by $B$ and let $L=\mathcal{O}_{\mathbb{P}^{n}}((d-1) r)$. Then $L$ is $d$-jet ample, but $\pi^{*} L$ fails to be that positive. The reason is that $L-q M$ fails to be $(d-q)$-jet ample for $q=d-1$. Indeed, as in the proof of the case 3 in Theorem 2.1 let $y \in R$ be a point on the ramification divisor $R \subset Y$ and $u_{1}, \ldots, u_{n}$ be local coordinates such that $R$ is locally defined by $u_{1}=0$. From the decomposition

$$
H^{0}\left(\pi^{*} L\right) \cong H^{0}\left(\mathcal{O}_{\mathbb{P}^{n}}((d-1) r)\right) \oplus u_{1} \cdot H^{0}\left(\mathcal{O}_{\mathbb{P}^{n}}((d-2) r)\right) \oplus \cdots \oplus u_{1}^{d-1} \cdot H^{0}\left(\mathcal{O}_{\mathbb{P}^{n}}\right)
$$

we infer that no section of $\pi^{*} L$ generates a jet of the form $u_{1}^{d-1} u_{i}$ for $i=2, \ldots, n$. In fact Theorem 2.1 implies that $\pi^{*} L$ is $(d-1)$-jet ample.

Remark 4.4. More generally, in the case of branched coverings, using the decomposition (1) one can show as in the previous example that if $L-q M$ fails to generate $(k-q)$-jets at the points of the branched locus, $\pi^{*} L$ fails to be $k$-jet ample.

Now we want to show that the assumptions of Theorem 3.1 cannot be weakened.

Example 4.5 (Geiser involution). Let $B \subset X \cong \mathbb{P}^{2}$ be a smooth plane quartic and let $\pi: Y \longrightarrow X$ be the double covering branched over $B$. Then $Y$ is the Del Pezzo surface of degree 2 and $-K_{Y} \cong \pi^{*} \mathcal{O}_{\mathbb{P}^{2}}(1)$, (see [7, 10.2.4]). Theorem 3.1 states that $-k K_{Y}$ is $k$-very ample for $k \geq 2$. This reproves a result of [16]. Moreover, in [16] it was shown that $-k K_{Y}$ is not $(k+1)$-very ample. This implies that our theorem is sharp.

Next, we want to apply our results to determine the dimension of certain linear systems of plane curves. Given general points $P_{1}, \ldots, P_{r} \in \mathbb{P}^{2}$ and an $r$-tuple $m=\left(m_{1}, \ldots, m_{r}\right)$ of integers, one is interested in the dimension, $l_{m}(d)$, of the linear system

$$
\left|\mathcal{O}_{\mathbb{P}^{2}}(d) \otimes \mathfrak{m}_{P_{1}}^{m_{1}} \otimes \cdots \otimes \mathfrak{m}_{P_{r}}^{m_{r}}\right|
$$

This problem has a long history; see [19] for a survey. The expected dimension, $e_{m}(d)$, of the above system is

$$
e_{m}(d)=\max \left(-1, \frac{d^{2}+3 d}{2}-\sum_{i=1}^{r} \frac{m_{i}\left(m_{i}+1\right)}{2}\right),
$$

where -1 is the dimension of the empty space. In other words, one expects that the conditions imposed by general "fat points" are independent. A natural question is whether $l_{m}(d)$ actually equals $e_{m}(d)$. This problem has been intensively explored recently. In particular one knows that $l_{m}(d)=e_{m}(d)$, if 
- $(d+3)^{2}>\frac{10}{9} \sum_{1}^{r}\left(m_{i}+1\right)^{2}(\mathrm{Xu}[22])$;

- $d \geq m_{1}+m_{2}+m_{3}$ and all $m_{i} \leq 4$ (Mignon [21]);

- $m_{1}$ arbitrary and $m_{2}=\cdots=m_{r} \leq 3$ (Ciliberto-Miranda [12]).

Here we show that a further result in this direction can be proved using the results of the present paper.

Proposition 4.6. Let $k \geq 2$ and

$$
m=(\underbrace{3 k+2, \ldots, 3 k+2}_{8}, k-2, k_{10}, \ldots, k_{r})
$$

with $\sum_{i=10}^{r} k_{i}=k+1$. Then for $d=9 k+6$ we have $l_{m}(d)=e_{m}(d)$.

Note that for numerical reasons none of the above criteria applies.

Proof. It is enough to show the proposition for points in a special position, since the function $l_{m}(d)$ is upper-semicontinuous in $\left(\mathbb{P}^{2}\right)^{r} \backslash$ diagonals and is at least $e_{m}(d)$. Then let $P_{1}, \ldots, P_{8}$ be in general position and let $P_{9}$ be the unassigned base point of $\left|\mathcal{O}_{\mathbb{P}^{2}}(3)-P_{1}-\cdots-P_{8}\right|$. Let $P_{9+1}, \ldots, P_{9+r}$ be any points distinct from $P_{1}, \ldots, P_{9}$. The assertion means that the ideal $I=\mathfrak{m}_{P_{1}}^{3 k+2} \otimes \cdots \otimes \mathfrak{m}_{P_{8}}^{3 k+2} \otimes \mathfrak{m}_{P_{9}}^{k-2} \otimes \mathfrak{m}_{P_{10}}^{k_{10}} \otimes \cdots \otimes \mathfrak{m}_{P_{r}}^{k_{r}}$ imposes independent conditions on the linear series $\left|\mathcal{O}_{\mathbb{P}^{2}}(d)\right|$. Hence it is enough to show that the mapping

$$
H^{0}\left(\mathcal{O}_{\mathbb{P}^{2}}(d)\right) \longrightarrow H^{0}\left(\mathcal{O}_{\mathbb{P}^{2}}(d) \otimes \mathcal{O}_{\mathbb{P}^{2}} / I\right)
$$

is surjective. This will follow (see [10]) if we show that the line bundle

$$
L=f^{*} \mathcal{O}_{\mathbb{P}^{2}}(d)-(3 k+2) E_{1}-\cdots-(3 k+2) E_{8}-(k-2) E_{9}
$$

is $k$-jet ample, where $f: X \longrightarrow \mathbb{P}^{2}$ is the blowing up of $\mathbb{P}^{2}$ in $P_{1}, \ldots, P_{9}$. Let $g: S \longrightarrow \mathbb{P}^{2}$ be the Del Pezzo surface obtained from $\mathbb{P}^{2}$ by blowing up $P_{1}, \ldots, P_{8}$. Then $\left|-K_{S}\right|$ has a base point $x=g^{-1}\left(P_{9}\right)$ and $\left|-2 K_{S}\right|$ is globally generated and defines a $2: 1$ mapping $p: S \longrightarrow Q$ onto a quadric cone $Q \subset \mathbb{P}^{3}$. It is known (cf. [7. 10.4.3]) that $p$ is branched over a smooth curve, the intersection of a cubic surface with $Q$, and the vertex $v=p(x)$ of $Q$. Let $\tau: \mathbb{F}_{2} \longrightarrow Q$ be the blowing up of $Q$ at $v$ with the exceptional divisor $D$ and let $\sigma: X \longrightarrow S$ be the blowing up of $S$ at $x$ with the exceptional divisor $E_{9}$. Then the following diagram

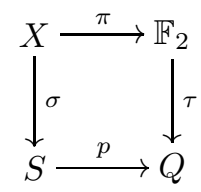

is commutative and $\pi$ is a double covering branched over a smooth divisor homologous to $2(2 D+3 F)$, where $F$ denotes the pull-back of the ruling on $Q$ by $\tau$. We observe that $L=-(3 k+2) K_{X}+(2 k+2) E=(3 k+2) \sigma^{*}\left(-K_{S}\right)-(k-2) E$, but since the jet ampleness of $-s K_{S}$ is not known, we cannot apply the results from [10] directly. On the other hand, $-(3 k+2) K_{X}+(2 k+2) E=(3 k+2) \pi^{*} F+(2 k+2) \pi^{-1}(D)$. This suggests we look for the positivity of the line bundles coming from $\mathbb{F}_{2}$ via $\pi$. We recall that a line bundle $L=a D+b F$ is $k$-jet ample on $\mathbb{F}_{2}$ (equivalently $k$-very ample, cf. e.g. [17, [8]) if and only if $L . D=-2 a+b \geq k$ and $L . F=a \geq k$. Then $H=(3 k+2) F+(k+1) D$ is $k$-jet ample and $H-M=(3 k-1) F+(k-1) D$ is $(k-1)$-jet ample. Then Theorem[2.1] implies that $L=\pi^{*} H$ is $k$-jet ample. 
Finally, we remark that the results of our paper can be applied both to show the positivity of certain line bundles, e.g. the jet ampleness of tensor powers of ample and spanned line bundles on Calabi-Yau varieties [11], and - what is even more interesting - to verify that certain line bundles are not $k$-jet or $k$-very ample; for instance, Proposition 5.2 of 4 follows immediately from Theorems 2.1 and 3.1 .

\section{Appendix A. Technical lemmas}

In this appendix we state and prove three lemmas needed in the course of the proof of Theorem 3.1 We begin with an elementary observation on local rings:

Lemma A.1. Let $(\mathcal{O}, \mathfrak{m})$ be a local ring, and let $I_{1}, \ldots, I_{\ell}, \ell \geq 2$, be ideals contained in $\mathfrak{m}$ such that length $\mathcal{O} / I_{1} \geq$ length $\mathcal{O} / I_{i}$ for all $i$. Let $K=\sum_{i=1}^{\ell}$ length $\mathcal{O} / I_{i}$. If $k<\infty$, then

$$
\text { length } \mathcal{O} / I_{2} \cap \cdots \cap I_{\ell} \leq \tau(K, \ell)
$$

Proof. We have

$$
\begin{aligned}
& \text { length } \mathcal{O} /\left(I_{2} \cap \cdots \cap I_{\ell}\right) \\
&=\text { length } \mathcal{O} / I_{2}-\text { length } \mathcal{O} /\left(I_{2}+I_{3} \cap \cdots \cap I_{\ell}\right)+\text { length } \mathcal{O} /\left(I_{3} \cap \cdots \cap I_{\ell}\right) \\
&= \ldots \\
&= {\left[\text { length } \mathcal{O} / I_{2}-\text { length } \mathcal{O} /\left(I_{2}+I_{3} \cap \cdots \cap I_{\ell}\right)\right]+\ldots } \\
& \quad+\left[\text { length } \mathcal{O} / I_{\ell-1}-\text { length } \mathcal{O} /\left(I_{\ell-1} \cap I_{\ell}\right)\right]+\text { length } \mathcal{O} / I_{\ell} \\
& \leq K-\text { length } \mathcal{O} / I_{1}-(\ell-2) .
\end{aligned}
$$

The assertion follows now from the fact that the length of $\mathcal{O} / I_{1}$ is at least $\lfloor K / \ell\rfloor+$ $1-\gamma(K, \ell)$.

The next two lemmas concern further properties of the function $\tau(\cdot, \cdot)$.

Lemma A.2. Let $\ell_{1}, \ldots, \ell_{m}, K_{1}, \ldots, K_{r}$ and $q$ be positive integers, and let $K=$ $\sum_{i=1}^{r} K_{i}$. If $\ell_{i} \geq 2$ for all $i$, then

$$
\sum_{i=1}^{m} \tau\left(K_{i}, \ell_{i}\right)+\sum_{i=m+1}^{r}\left\lfloor\frac{K_{i}}{q+1}\right\rfloor \leq \max \left\{\tau(K, \ell) \mid \min \left(\ell_{1}, \ldots, \ell_{q}, q+1\right) \leq \ell\right\} .
$$

Proof. First we note that it is enough to prove

$$
\sum_{i=1}^{m} \tau\left(K_{i}, \ell_{i}\right)+\sum_{i=m+1}^{r}\left\lfloor\frac{K_{i}}{q+1}\right\rfloor \leq \tau(K, \ell)
$$

with $\ell=\max \left(\ell_{1}, \ldots, \ell_{m}\right)$. Due to the fact that

$$
\left\lfloor\frac{K_{i}}{q+1}\right\rfloor \leq K_{i}-\left\lfloor\frac{K_{i}}{2}\right\rfloor-1+\gamma\left(K_{i}, 2\right)=\tau\left(K_{i}, 2\right)
$$

we may assume $r=m$ (by setting $l_{i}=2$ for $m<i \leq r$ ). Then (8) reads $\sum_{i=1}^{r} \tau\left(K_{i}, \ell_{i}\right) \leq \tau(K, \ell)$ and it suffices to prove it for $r=2$. We may also assume $\ell_{1} \leq \ell_{2}=\ell$. From

and

$$
\left\lfloor\frac{K_{1}}{\ell_{1}}\right\rfloor \geq\left\lfloor\frac{K_{1}}{\ell}\right\rfloor+\gamma\left(K_{1}, \ell_{1}\right) \cdot \min \left(1, \ell-\ell_{1}\right)
$$

$$
\left\lfloor\frac{K_{1}}{\ell}\right\rfloor+\left\lfloor\frac{K_{2}}{\ell}\right\rfloor \geq\left\lfloor\frac{K}{\ell}\right\rfloor-1+\gamma\left(K_{1}, \ell\right)+\gamma\left(K_{2}, \ell\right)-\gamma\left(K_{1}, \ell\right) \cdot \gamma\left(K_{2}, \ell\right)
$$


we get

$$
\begin{aligned}
& \tau\left(K_{1}, \ell_{1}\right)+\tau\left(K_{2}, \ell_{2}\right) \leq K-\left\lfloor\frac{K}{\ell}\right\rfloor-\ell+\gamma\left(K_{1}, \ell\right) \cdot \gamma\left(K_{2}, \ell\right)+1 \\
& +\left[2-\ell_{1}\right]+\left[\gamma\left(K_{1}, \ell_{1}\right)-\gamma\left(K_{1}, \ell\right)-\gamma\left(K_{1}, \ell_{1}\right) \cdot \min \left(1, \ell-\ell_{1}\right)\right] .
\end{aligned}
$$

Now the assertion follows by observing that $\gamma\left(K_{1}, \ell\right) \cdot \gamma\left(K_{2}, \ell\right) \leq \gamma(K, \ell)$ and that the terms in the square brackets are non-positive.

Lemma A.3. Let $a \geq b \geq 2$ be positive integers. Then $\left\lfloor\frac{a}{b}\right\rfloor \leq \tau(a, b)$.

Proof. Since $2\left\lfloor\frac{a}{b}\right\rfloor-\gamma(a, b) \leq 2 \frac{a}{b}-1$, we have

$$
\left\lfloor\frac{a}{b}\right\rfloor-\tau(a, b) \leq b+2 \frac{a}{b}-a-2 .
$$

Let $f(b)=b^{2}+2 a-a b-2 b$. It is now enough to show that $f(b) \leq 0$ for $b$ in the given range. Since $f$ is a quadratic function it is in fact enough to check that $f(2) \leq 0$ and $f(a) \leq 0$, which is obvious.

\section{REFERENCES}

[1] Barth, W., Peters, C., Van de Ven, A.: Compact complex surfaces. Ergeb. Math. Grenzgeb. (3) 4, Springer-Verlag, 1984. MR 86c:32026

[2] Bauer, Th., Szemberg, T.: Higher order embeddings of abelian varieties. Math. Z. 224, 449455 (1997) MR 98a:14009

[3] Bauer, Th., Szemberg, T.: Primitive higher order embeddings of abelian surfaces. Trans. Amer. Math. Soc. 349, 1675-1683 (1997) MR 97h:14005

[4] Beltrametti, M.C., Di Rocco, S., Sommese, A.J.: On higher order embeddings of Fano threefolds by the anticanonical linear system. J. Math. Sci. Univ. Tokyo, 5, 75-97 (1998). MR 99d:14036

[5] Bauer, Th., Di Rocco S., Szemberg, T.: Generation of jets on K3 surfaces. J. Pure Appl. Algebra, 146, 17-27 (2000). CMP 2000:07

[6] Beltrametti M.C., Francia P., Sommese A.J.: On Reider's method and higher order embeddings. Duke Math. J. 58, 425-439 (1989) MR 90h:14021

[7] Beltrametti, M.C., Sommese, A.J.: The Adjunction Theory of Complex Projective Varieties. Expositions in Mathematics, 16, Walter de Gruyter, Berlin (1995). MR 96f:14004

[8] Beltrametti, M.C., Sommese, A.J.: On the preservation of $k$-very ampleness under adjunction. Math. Z. 212, 257-283 (1993) MR 94a:14002

[9] Beltrametti, M.C., Sommese, A.J.: On $k$-jet ampleness. Complex analysis and geometry (Ancona, Vincenzo, eds.), New York, Plenum Press, 1993, pp. 355-376. MR 94g:14006

[10] Beltrametti, M.C., Sommese, A.J.: Notes on embeddings of blowups, J. Algebra 186, 861-871 (1996) MR 97m:14004

[11] Beltrametti, M.C., Szemberg, T.: On higher order embeddings of Calabi-Yau threefolds, preprint

[12] Ciliberto, C., Miranda, R.: Degenerations of planar linear systems, J. Reine Angew. Math. 501, 191-200 (1998) CMP 98:16

[13] Debarre, O., Hulek, K., Spandaw, J.: Very ample linear systems on abelian varieties. Math. Ann. 300, 181-202 (1994) MR 95k:14065

[14] Demailly, J.-P.: Singular Hermitian metrics on positive line bundles. Complex algebraic varieties (Bayreuth, 1990), Lect. Notes Math. 1507, Springer-Verlag, 1992, pp. 87-104. MR 93g:32044

[15] Demailly, J.-P.: $L^{2}$ vanishing theorems for positive line bundles and adjunction theory. In: Transcendental methods in algebraic geometry (Cetraro, 1994), Lect. Notes Math. 1646, Springer-Verlag, 1996, pp. 1-97. MR 99k:32051

[16] Di Rocco S.: $k$-very ample line bundles on Del Pezzo surfaces. Math. Nachr. 179, 47-56 (1996) MR 97h:14007

[17] Di Rocco S.: Generation of $k$-jets on toric varieties. Math Z. 231, 169-188 (1999) CMP 99:14 
[18] Geramita, A. V., Gimigliano G., and Harbourne B.: Projectively normal but superabundant embeddings of rational surfaces in projective space. J. of Algebra 169, 791-804, (1994). MR 96f:14044

[19] Gimigliano, A.: Our thin knowledge of fat points. The Curves Seminar at Queen's, Vol. VI (Kingston, ON, 1989), Exp. No. B, 50 pp., Queen's Papers in Pure and Appl. Math., 83, Queen's Univ., Kingston, ON, 1989. MR 91a:14007

[20] Lazarsfeld, R.: Lectures on linear series. Park City/IAS Mathematics series vol. 3, 161-219 (1993) MR 98h:14008

[21] Mignon, T.: Systèmes de courbes planes à singularités: le cas des multiplicités inférieures ou égales à quatre. Preprint

[22] Xu, G: Ample line bundles on smooth surfaces. J. Reine. Angew. Math. 469, 199-209 (1995) MR 96k:14003

Mathematisches Institut, Universität Erlangen-Nürnberg, Bismarckstrasse $1 \frac{1}{2}$, D91054 Erlangen, Germany

Current address: Fachbereich Mathematik und Informatik, Philipps-Universität Marburg, Hans-Meerwein-Straße, D-35032 Marburg, Germany

E-mail address: tbauer@mathematik.uni-marburg.de

KTH, Department of Mathematics, S-100 44 Stockholm, Sweden

Current address: Department of Mathematics, Yale University, PO Box 208229, Hillhouse Ave. 70, New Haven, Connecticut 05620-8229

E-mail address: sandra.dirocco@math.yale.edu

Instytut Matematyki, Uniwersytet Jagielloński, Reymonta 4, PL-30-059 Kraków, POLAND

Current address: Department of Mathematics, University of Michigan, Ann Arbor, Michigan 48109

E-mail address: szemberg@math.1sa.umich.edu 